\section{Train in non-invasive aesthetic} rehabilitation

Join Rafael Beolchi (pictured) on 28 November at the Woburn House Conference Centre, London, for 'Hands-On With Ultradent', a full day course on choosing and individualising materials for non-invasive aesthetic rehabilitation. You will be able to discuss available techniques with like-minded individuals and discover advanced aesthetic procedures with dental composites. This will allow you to identify which materials would be able to reproduce even the smallest anatomical and optical details of the natural tooth. The day includes a hands-on session for you to put what you've learnt into practice, and also a session on whitening protocols for different patients. It is free to attend.

For more information visit handsonwithultradent.eventbrite.co.uk or call 01634877599 .

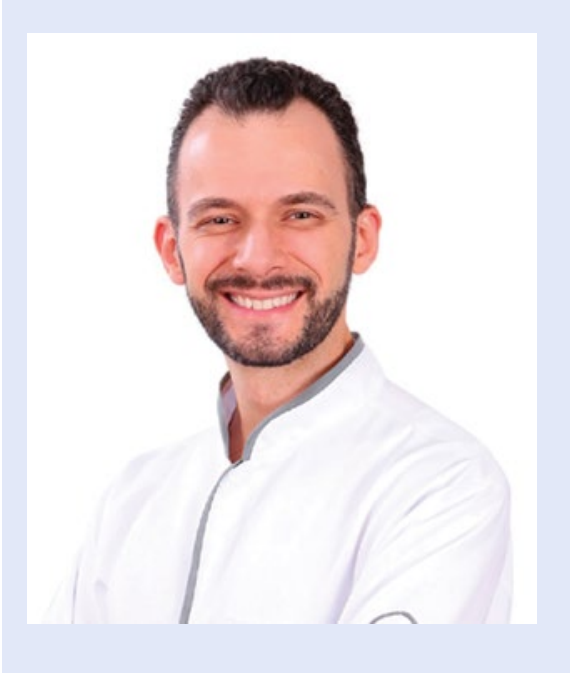

\section{Excellent resistance to cyclic} fatigue

EdgeEndo, one of the world's largest $\mathrm{NiTi}$ rotary file suppliers, showcased its endodontic solutions at the $19^{\text {th }}$ Biennial Congress of European Society of Endodontology (ESE) in Vienna in September. The ESE is a federal organisation representing national endodontic and dental societies throughout Europe and has 36 member societies from 33 European countries. It represents over 7,500 ordinary members and over 500 individual specialist, certified, guest and postgraduate members.

Amongst the high-quality EdgeEndo product lines presented at the Congress were NiTi files, carrier based obturators, and points. The files and obturators are excellent alternatives to the current products on the market and can be used with most existing endodontic motors and obturator ovens in the practice. The products are available through Henry Schein.

Edge NiTi files offer excellent resistance to cyclic fatigue, and enhanced flexibility that allow clinicians to do high-quality root canals in an efficient manner, all for a reasonable cost.

More information about the EdgeEndo product line is available through your local Henry Schein representative.

\section{Atraumatic tooth extraction}

Manufacturer of dental consumables

Directa, which has its HQ in Sweden, has acquired extraction instrument line Physics Forceps from Detroit based manufacturer Golden Dent.

Physics Forceps instruments are now included in the Directa product range. The acquisition strengthens Directa's supply of atraumatic extraction instruments and complements the Luxator instrument line.

Designed and patented in 2007 by Dr Richard Golden, the Physics Forceps atraumatic extraction system is among the most clinically effective and easy to use extraction systems on the market. The Physics Forceps operate as an elevator, rather than forceps, using first class lever mechanics. Placing a constant and steady load on the tooth, allowing 'creep' to build, releasing hyaluronic acid, resulting in the breakdown of the periodontal ligaments. It is an atraumatic method of extracting teeth regardless of the condition of the tooth, while providing a positive patient experience.

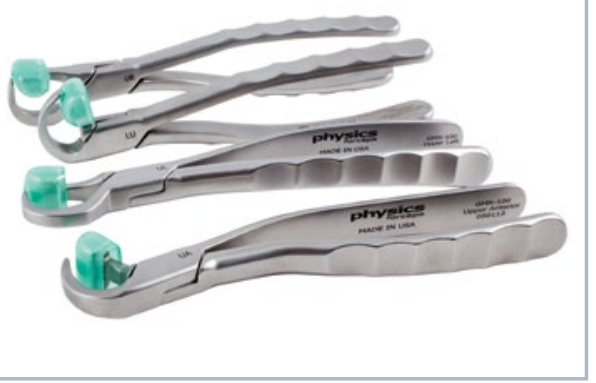

\title{
British made implants celebrate 20 years
}

On 28 September OsteoCare celebrated its 20-year anniversary at the Brooklands museum.

Many of OsteoCare's dentists and suppliers attended the event to commemorate 20 years of success. The day consisted of free CPD verified seminars, freedom to explore the museum and then a celebratory afternoon tea.

The seminars covered 'Digital Dentistry' by Dr Martin Attariani, 'Increasing bone volume in implant placement' by Professor
Amr Zahran and 'No implantology without Periodontology (NIWOP)' by Dr Kashif Hafeez.

Brooklands Museum was the perfect place to celebrate the 20-year anniversary as it is the birthplace of British motorsport and aviation which ties into OsteoCare's heritage. OsteoCare are the pioneers of products such as the centre finder and one of the first companies in the UK to produce mini/midi implants and conical shaped implants. As the only British made dental implant system OsteoCare aims to continue to offer dentists a simplified system and excellent quality and value products. It will continue this exciting journey with the invention of new products and new courses to fulfil the dentist's needs.

OsteoCare's 20-year journey has definitely been memorable and the company would like to thank its users for becoming part of that journey.

To find out more information about future courses visit http://osteocare. uk.com/training-education/. 